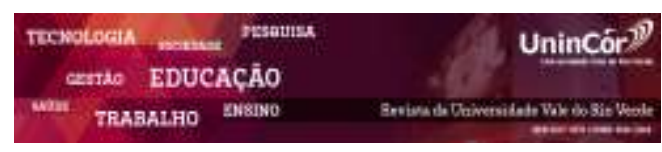

Revista da Universidade Vale do Rio Verde ISSN: 1517-0276 / EISSN: 2236-5362 v. 17 | n. 1 | Ano 2019

\begin{abstract}
Aldair Fernandes Silva Instituto Federal de Minas Gerais - IFMG aldairh10@gmail.com
\end{abstract}

Ricardo Soares de Melo Centro Universitário Internacional - Uninter rikardo@gmail.com

Ítalo Brener de Carvalho Centro Federal de Educação Tecnológica de Minas Gerai s - CEFETMG italobrener@hotmail.com

\section{ENSINO - APRENDIZAGEM: OS \\ CONCEITOS DO LAZER PARA A FORMAÇÃO E MUDANÇA DE COMPORTAMENTO EM AMBIENTES EDUCACIONAIS}

\section{RESUMO}

Sugerindo buscar possibilidade de se mensurar o impacto do lazer em ambientes de formação, na profissionalização, no ensino e educação este artigo teórico discute um tema no limite entre tres áreas científicas: o lazer, o trabalho e a gestão de aprendizagem. As metodologias utilizadas na aprendizagem de competências laborais se desenvolvem desde mudanças sociais ocorridas por meio da revolução industrial até os dias de hoje, se relacionando fortemente com as capacidades adquiridas durante o processo do aprendizado, da formação de Competências, de Habilidade e de Atitudes. De forma bivalente: (1) o comportamento social no trabalho e fora dele, são impactados pelas consequências do trabalho alienado, das longas jornadas de trabalho e dos novos processos de urbanização. A perspectiva do lazer utilizada neste estudo contribui em aspectos que direcionam o aprendiz, o individuo em formação, na compreensão das relações de trabalho mais justas, relacionados principalmente, na melhoria do bemestar em níveis individuais, comunais e até mesmo globais em ambientes laborais. Mas também em outra perspectiva a de que (2) o Lazer e seu papel na gestão, evocado por uma perspectiva funcionalista, contribui como catalizador da formação do comportamento, na preparação para o trabalho, como ferramenta utilizada durante o processo formativo de que prepara o individuo para o trabalho. Ao final desta discussão, apresenta-se cinco pressupostos como sugestões de pesquisas necessárias para o desenvolvimento de conceitos basilares à esta discussão.

Palavras-chave: Aprendizagem, Lazer, Ensino, Mudanças de Comportamento.

\section{TEACHING - LEARNING: THE CONCEPTS OF LEISURE FOR THE FORMATION AND CHANGE OF BEHAVIOR IN EDUCATIONAL ENVIRONMENTS}

\begin{abstract}
Suggesting the possibility of measuring the impact of leisure in training environments, professionalization, teaching and learning focusing on the job skills in the learning tool. This theoretical article discusses a theme in the limit between three scientific areas: leisure, work and learning. Faced with studies of the social changes that have taken place since the industrial revolution to the present, strongly related to the Skills and Attitudes during the process of learning management. In a bivalent way: (1) social behavior in and out of work, still as a consequence of alienated work, long working hours and new urbanization processes. Structured as time antagonistic to the time of productive work, the perspective of leisure contributes to fairer working relations with
\end{abstract}


goals directed towards new paradigms, related mainly, in the improvement of well-being at individual, communal and even global levels in work environments. And (2) Leisure and its role in management, evoked by a functionalist perspective, which contributes as a catalyst for the formation of behavior. At the end of this discussion, it presents basic assumptions and concepts to this discussion.

Keywords: Learning, Work, Leisure, Teaching ,Behavior Change.

\section{Recebido em: 05/12/2018 - Aprovado em: 13/04/2019 - Disponibilizado em: 15/07/2019}

\section{INTRODUÇÂO}

Avaliar os aspectos do bem-estar na sociedade, como exemplos da promoção da saúde física (objetiva e percepções subjetivas), da saúde mental (por exemplo, a resiliência, estresse e Burnout), da discriminação, da marginalização, de inclusão, de acesso, da capacitação e da possibilidade de diminuir disparidades (Anderson, Ostrom, Corus, Fisk, Gallan, Giraldo, Mende, Mulder, Rayburn, Rosenbaum, Shirahada, Williams, 2013; Rosenbaum, Corus, Ostrom, Anderson, Fisk, Gallan, Williams, 2011) são temas de intersecção entre pesquisas de lazer e de Educação.

Este ensaio, com características metodológicas exploratórias, parte de um princípio: o caráter transformativo de serviços ligados a propostas de bem-estar (Anderson, 2010) com foco na construção de mudanças edificantes que melhorem a vida de indivíduos, de famílias, da sociedade e dos sistemas que interligam agentes e consumidores (Anderson et. $a l, 2013)$.

Apesar da Lógica de Mercado se concentrar em preâmbulos lucrativos, postular que o valor percebido por qualquer público-alvo é em sua essência moduladas por variáveis como: o tempo, renda, aspectos sociais, instituições, símbolos, inseridos em uma lógica de política pública, bem como de atividades ligadas a características psicológicas individuais. É possível apontar, que o cotidiano seria a célula das transformações almejadas pela sociedade. E uma vez centrada no trabalho a experiência cotidiana humana, é apreendida, é compartilhada, é elaborada e negociada (Bauman, 2009) pelo processo EnsinoAprendizagem.

Leite (2012, p. 14) em sua abordagem sobre sistemas inteligentes, destaca que as cidades, suas instituições e meios se reinventam, "elas não são fossilizadas: as melhores cidades são aquelas que continuamente sabem se renovar, funcionam similarmente a um organismo - quando adoecem, se curam mudam". Da mesma forma, a sociedade contemporânea urge por novos modelos educação, por novos modelos e metodologias, mais justos e sustentáveis. Ou seja, transformar a rede de preparação e formação de indivíduos com redes estratégicas que impactem no aprimoramento, do desenvolvimento e crescimento do indivíduo e consequentemente de uma sociedade.

Originalmente constituído por propostas que buscam proposições positivas de melhoria da 
qualidade de vida, as abordagens do Lazer são fortemente criticadas. Relata-se que organizações e instituições não corroboram, com o aprendizado de seus colaboradores, ignoram ou até mesmo prejudicam o sistema produtivo com politicas predatórias e degradantes. No aspecto qualitativo do lazer como prática pedagógica, Marcellino (2010) classifica o lazer como um recurso de ajuste das pessoas à sociedade, ou seja, de modo a manter a ordem social já estabelecida. Para o mesmo autor o lazer oferece oportunidades de contato, percepção e reflexão sobre as pessoas e as realidades onde estão inseridas. Além disso, é um espaço para participação cultural, possibilitando a instauração de uma nova ordem social, levando os sujeitos a uma formação integral, transformando-os em cidadãos, atuando em sua cultura, modificando a sociedade.

O desafio de pesquisas propositivas é oportuno. Sendo um desafio propor métricas acadêmicas de investigações que promovam este avanço e que promovam uma atuação conjunta entre a sociedade civil organizada, do setor corporativo e, obviamente, da atuação das instituições de educação e formação como gargalo em países como o Brasil.

Assim este artigo tem como objetivo buscar compreender o lazer como agente catalizador no processo formativo, como metodologia que privilegie a aprendizagem fornecendo no processo de ensino a melhoria do bem-estar, dos processos de mudança de comportamento profundamente ligados as questões Sociais, de Educação e de Formação Cidadã.

\section{AGENTE TRASFORMADOR}

Vários estudos em Lazer apontam a obra de Acácio Ferreira (1959) como uma obra pioneira neste campo. Em sua obra entitulada "Lazer Operário", Ferreira afirma que a compreensão lazer (apesar de ser um conceito nesta ótica generalizado) como uma fração de tempo, que em contraponto com o tempo trabalhado é nominado como tempo livre. Dessa maneira a oposição entre Trabalho e Lazer, mesmo possuindo, características distintas, integram a mesma dinâmica social em um sistema cotidiano de vida e vivência (WERNECK, 2000) estabelecendo relações dialéticas e também antagônicas.

Nesta perspectiva o lazer é compreendido como uma conquista do trabalhador que portanto que só seria oportunizado no contraponto, entre, o tempo dedicado no ambiente de trabalho e o tempo restante. Esta perspectiva traz por si a variável do "tempo" como um marco de significado regulatório. Por exemplo: envolve a quantidade de horas trabalhadas no dia, "O Estado, com a evolução das leis trabalhistas, veio paulatinamente reduzindo a jornada de trabalho para 12, 10, 9 e 8 horas de labor, aumentando os momentos de folga, e, desta forma, criando o problema do uso adequado das horas de lazer" (Marinho, 1957, p.135). Outro exemplo, o tempo que envolve o período de férias, os dias dedicados aos descansos semanais, que regulamentados pelas leis trabalhistas, são remuderados. "O período entre duas jornadas consecutivas de trabalho e os repousos obrigatórios, isto é, o descanso semanal e as férias anuais" (Sussekind, Marinho \& Góes 1952, p.16-17). 
A ideia de que as horas de lazer deveriam ser preenchidas apenas com as atividades recreativas consideradas, pelos segmentos dominantes no mercado capitalista, como horas saudáveis (Gomes, 2014). Percebe-se que uma pesquisa com uma ótica voltada para o lazer é fundamentada na perspectiva do lazer complementar as horas de trabalho, e na proposta de criar atividades físicas que fortaleçam a força de trabalho. Ou seja mesmo no tempo de lazer, que este tempo fortaleça a força de trabalho, habilitando-a para trabalhar de forma saudável, melhor ou mais.

No entanto, é com Dumazedier (1973) possibilita questionar esta compressão. $\mathrm{O}$ autor afirma que a compreensão restrita ao lazer com variável modelada e impactada pelo "tempo" subtraído da jornada de trabalho, torna-se crítica. Não menos imporatne mas ampliando estes conceitos o autor amplia a discussão propondo novoas variáveis que podem ser uincluidad nesta reflexão que teriam para além do "tempo" premissas como:

(1) caráter liberatório: o lazer é liberação de obrigações institucionais (profissionais, familiares, sócio espirituais e sócio-políticas) e resulta de uma livre escolha.

(2) caráter desinteressado: o lazer não está, fundamentalmente, submetido a fim algum, seja lucrativo, profissional, utilitário, ideológico, material, social, político, sócios spiritual.

(3) caráter hedonístico: o lazer é marcado pela busca de um estado de satisfação, tomado como um fim em si: "isso me interessa". Essa busca pelo prazer, felicidade, alegria ou fruição é de natureza hedonística e representa a condição primeira do lazer.
(4) caráter pessoal: as funções do lazer (descanso, divertimento e desenvolvimento da personalidade) respondem às necessidades do indivíduo, face às obrigações primárias impostas pela sociedade.

A obra de Dumazedier (1973) proporciona a amplitude do conceito de lazer, que pode portanto ser compreendido como o conjunto de ocupações, que de livre vontade, a qualquer indivíduo, possa ser utilizada tanto para o repouso, para divertir-se, para recrear-se ou ainda que por meio dela desenvolve sua formação desinteressada, sua participação social voluntária, ou a livre criatividade, excluindo as obrigações sejam profissionais, familiares ou sociais.

Fundamentando-se no pensamento de Dumazedier, Renato Requixa e Luiz Octávio Camargo também reforçam as concepções de lazer que coincidem com os postulados do sociólogo francês Requixa (1980, p.35) que define o lazer como "ocupação não obrigatória, de livre escolha do indivíduo que a vive, e cujos valores propiciam condições de recuperação psicossomática e de desenvolvimento pessoal e social."

Esta perspectiva possibilita uma inversão de ótica: do lazer do contraponto ao trabalho, para o lazer como um direito, como agente de formativo da cidadania e como dimensão da vida e cotidiano humano no desenvolvimento de sociedades.

\section{AGENTE DE FORMADOR}

Nicanor Miranda no ano de 1935, também já havia destacado a variável "tempo" como moduladora do lazer. Em sua concepção o tempo do lazer deveria ser corretamente preenchido, as 
horas vagas da população trabalhadora deveria ser usada para o aprendizado.

Relatado por Gomes (2003) o texto de Miranda discorria sobre a formação de uma jovem moça operária. Destacando variáveis inseridas no contexto. $\mathrm{O}$ autor ressalta que devido a baixa escolaridade, devido a uma educação incompleta, as jovens (se referindo diretamente a força de trabalho feminina) seriam incapazes de preencher o tempo de lazer de forma organizada e proveitosamente educativa. Percebe-se nesta referência a justificativa que permite portanto a relação entre a variável tempo e educação.

Marcelino (2009), também resgata aspectos construtivos pedagogicamente analisados, destacando os seguintes aspectos educativos do lazer: educação para o lazer e pelo lazer - (1) enquanto objeto [...], na perspectiva de chamar a atenção para a importância do lazer na nossa sociedade [...] e dando iniciação ao conteúdo cultural específico (físico-esportivo), mostrando a relação com os demais; (2) enquanto veículo [...], trabalhando os conteúdos vivenciados pelo lazer, [...] buscando a superação do conformismo, pela crítica e criatividade; e (3) enquanto conteúdo e forma, no desenvolvimento das aulas, buscando incorporar, o máximo possível, o componente lúdico da cultura.

Este mesmo autor intitulou "pedagogia da animação" a relevância do construto lazer, escola e processo educativo para a aprendizagem. Marcelino (2011) vê essa pedagogia como embasadora de uma nova prática educativa e realimentada através dessa própria prática, considerando as possibilidades do lazer como canal viável de atuação no plano cultural, de modo integrado com a escola. Dessa forma, o lazer poderia contribuir para a elevação do senso comum, numa perspectiva de transformação da realidade social, sempre em conexão com outras esferas de atuação política.

Apesar da divisão do trabalho ter implicado na especialização de tarefas, ou seja, no avanço de habilidades por meio da especialização, por sua vez, gerou um refinamento dos recursos operantes, nas pessoas, em suas habilidades e conhecimento. Neste contexto, é preciso desenvolver conhecimentos e habilidades através da aprendizagem organizacional para "encontrar, extrair, cultivar, inventar, fabricar e usar recursos operados; ou seja, os benefícios derivam da aplicação de recursos operantes a recursos operados", de acordo com Vargo e Lusch (2006).

\section{ABORDAGEM FUNCIONALISTA}

Como visto nos parágrafos anteriores autores como Nelson Marcellino vêm sendo consideravelmente citado nos estudos sobre o lazer em nosso país. Em seu livro Lazer e humanização, observa-se que sua produção intelectual também endossou as ideias do também referenciado autor, Dumazedier. Embora as análises sejam redimensionadas em alguns pontos são fundamentados por subsidiar considerações marxistas, e portanto possível de serem abordados por "abordagens funcionalistas" do lazer.

Tais abordagens são conservadoras, disciplinadoras, visam à manutenção do status quo, mascaram injustiças sociais e funcionam como válvulas de escape das tensões. Como as 
abordagens funcionalistas as perspectivas do processo Ensino-Aprendizado buscar-se-ia o ajuste do indivíduo, de forma acrítica ao contexto em que vive, incentivando o consumismo em relação ao lazer.

Ao redimensionar o lazer como cultura, esta compreensão supera o seu entendimento como mero "conjunto de ocupações". Mesmo promovendo um avanço na compreensão de lazer, alguns pontos do conceito de Marcellino também vêm sendo foco de questionamentos. Vânia Noronha Alves (2003, p.98) assinala que, apesar da apropriação, por muitos, desta concepção de lazer, algumas questões precisam ser repensadas: "talvez, fosse mais correto falar em tempo disponível, ao invés de tempo livre" evoluindo para conceitos como tempo conquistado de Bramante (1998), Segundo sua interpretação, o tempo é um "conceito objetivamente inelástico" que vem sendo encarado como uma "mercadoria" de luxo, em que a máxima "tempo é dinheiro" chega a refletir o seu significado. "Portanto, 'conquistar' um tempo da não obrigação vem se impondo como um desafio para todos que desejam exercitar a face humana da vida plena" (1998, p.11).

Marcellino (1987, p.29) afirma que: “o lazer se traduz por uma dimensão privilegiada da expressão humana dentro de um tempo conquistado, materializada por meio de uma experiência pessoal criativa, de prazer e que não se repete no tempo/espaço, cujo eixo principal é a ludicidade. [...]" e portanto sendo possível mais uma vez incluir esta temática no processo formativo do indivíduo.

Trilhando os caminhos marxistas e assim possibilitando uma analise funcionalista
Mascarenhas (2001, p.92) também formulou um conceito de lazer, conforme suas palavras, “o lazer se constitui como um fenômeno tipicamente moderno, resultante das tensões entre capital e trabalho, que se materializa como um tempo e espaço de vivências lúdicas, lugar de organização da cultura, perpassado por relações de hegemonia."

Assim, o lazer é uma dimensão da cultura construída socialmente, em contexto especifico, a partir de a principio, quatro elementos interrelacionados:

(1) tempo, que corresponde ao usufruto do momento presente e não se limita aos períodos institucionalizados para o lazer (final de semana, férias, etc.);

(2) espaço-lugar, que vai além do espaço físico por ser um "local" do qual os sujeitos se apropriam no sentido de transformá-lo em ponto de encontro (consigo, com o outro e com o mundo) e de convívio social para o lazer;

(3) manifestações culturais, conteúdos vivenciados como fruição da cultura, seja como possibilidade de diversão, de descanso ou de desenvolvimento;

(4) ações, que são fundadas no lúdico entendido como expressão humana de significados $\mathrm{da} / \mathrm{na}$ cultura referenciada no brincar consigo, com o outro e com a realidade.

Como constituem elementos interrelacionados, integrados considera-se que não há impedimento para a execução destas por parte de empresas privadas por agentes produtivos de diferentes portes, contribuindo para um desdobramento e impacto no bem-estar das ações de gestão organizacional. 


\section{LAZER E SUA CAPACIDADE DE CONTRIBUIR PARA O BEM ESTAR LABORAL}

Desde os primórdios da humanidade, os seres humanos se esforçado para melhorar o seu bem-estar e, consequentemente, a qualidade de suas vidas. E são os acessos a serviços ao longo da vida que geram Bem-estar (Ostrom et. al., 2010). Veja bem, se o bem-estar está relacionado com qualquer nível de satisfação das necessidades básicas através (Maslow, 1943 e 1987) as necessidades de água, comida, abrigo, status e reconhecimento, podem ser atendidas por meio dos serviços de distribuição de água, pela disponibilidade de alimentos pelos supermercados, meios de hospedagem e construção civil ou reformas, por serviços de redes sociais.

As metas e objetivos deste novo paradigma se relacionam principalmente na melhoria do bem-estar em níveis individuais, comunais e até mesmo globais. Observa-se que o direcionamento de pesquisas podem seguir as seguintes agendas: (1) bem-estar, incluem questões de qualidade de vida. (2) serviços que já possuem qualidades transformadoras pela sua concepção inerente e são destinadas a melhorar o bem-estar (mas na realidade não o fazem) e (3) serviços que não foram concebidos com qualidades transformadoras mas que poderiam melhorar não intencionalmente o bem-estar.

O termo ecossistema é escolhido por indicar uma dinâmica de adaptação constante do sistema. Como os sistemas são auto ajustáveis, simultaneamente funcionando e reconfigurando o processo de integração de recursos e criação de valor. Uma visão de sistema difere da visão de rede pelo fato de que no sistema, a cada integração de recurso, provisão de serviço e criação de valor, ocorre uma mudança em certo grau do contexto para a próxima iteração e determinação da criação de valor (Wieland et. al., 2012) .

A tentativa de modificar as atitudes ou o comportamento são segundo Kotler (1978, p.293), os seguintes tipos:

(1) mudança cognitiva: Há muitas causas sociais cujo propósito é informar a opinião pública, alterando, assim, seu nível de conhecimento a respeito de determinados assuntos. Ao identificar os grupos mais carentes de informações, ao identificar hábitos e as predisposições comportamentais de modo a subsidiar a elaboração das mensagens; Exemplos: programa para explicar o valor nutritivo de alimentos; programa para chamar a atenção sobre o problema da velhice.

(2) mudança de ação: Outro tipo de causa social é aquela que visa alterar a atitude das pessoas, tentando, frequentemente, induzir um número máximo dessas pessoas a realizar uma ação específica, durante certo período. Este tipo de mudança apresenta maior grau de complexidade em relação à mudança cognitiva. O aprendizado para a execução de alguma coisa e isto envolve um custo para a pessoa. Mesmo que a sua predisposição seja favorável ela terá que ser deslocada da inércia. Exemplos: programa de vacinação em massa; programa para atrair doadores de sangue; programa para a prevenção do câncer; mutirão para a limpeza da rua.

(3) mudança no comportamento: Aumenta o grau de complexidade em relação às 
mudanças anteriores. Algumas causas sociais têm por objetivo ajudar as pessoas a modificar algum aspecto de seu comportamento, tendo em vista o bem-estar individual. A mudança comportamental lida com dinâmicas psicológicas mais arraigadas, requerendo, portanto, mais habilidades na sua condução. Exemplos: programas para desencorajar o vício do fumo; programas contra entorpecentes, bebidas alcoólicas, etc.

(4) mudança de valor: As causas que procuram alterar crenças ou valores lidam com o tipo de mudança de maior grau de complexidade. Exemplo: programa contra a segregação racial. Kotler (1978, p.150).

\section{CONSIDERAÇÕES FINAIS}

Este artigo teórico discutiu um tema pertinente a duas áreas científicas, a intersecção entre pesquisas de lazer e de pesquisas em gestão, apresentando uma proposta de evolução do pensamento de como o Lazer pode ser taxado como Catalizador na Formação do Comportamento, através da aprendizagem.

$\mathrm{O}$ ambiente de trabalho, a vida para além do tempo laboral envolve aspectos de bem-estar. Tanto a saúde física como a saúde mental, a inclusão, o acesso, a capacitação e a possibilidade de diminuir disparidades, de direito e de realização humana focam na formação de possíveis mudanças, edificantes, que possibilitam um comportamento social no trabalho e fora dele, que melhorem a vida de indivíduos, de famílias, da sociedade e dos sistemas que interligam seus agentes.

Baseado na melhoria do bem-estar por meio da mudança de comportamento e pela criação de valor, o lazer e a gestão contribuem para relações de trabalho um pouco mais justas com metas direcionadas para novos paradigmas.

O Lazer e seu papel na gestão, evocado por uma perspectiva funcionalista, contribui como catalizador da formação do comportamento quanto maior o nível de atividades do indivíduo maior a integração dos recursos e co-criação de valor.

Aceitação dos valores, que tornam a opção aceitável para adoção, empenho e aplicação de recursos individuais que permitam um nível alto de engajamento dependem dos valores sejam simbolicamente negativos para o indivíduo.

Influenciam na percepção da opção como relevante para si, para familiares e para a sociedade, já que instituições que sentem que valor atribuído a uma proposta de valor, podem compreender que quanto mais as regras institucionais restringirem o campo de alternativas, mais severas as sanções e mais eficazes os mecanismos de garantia, menor a possibilidade de o indivíduo perceber a mudança como relevante.

Mesmo utópico as características individuais também podem influenciar, por exemplo, o nível de emancipação e capacidade crítica de uma sociedade, quanto maior o nível de emancipação, mais recursos são integrados.

Uma lógica institucional ou da simbólica determina o que é positivo e o que é negativo, contraposta ao nível de emancipação determina quais recursos serão integrados. Pode ocorrer também a intervenção do grupo de amigos e familiares, que representam recursos individuais. Quanto maior o número de interações com o 
grupo, maior o número de recursos integrados e a co-criação de valor na mudança.

Ao contrário atividades como exemplificadas de atitude positiva, pró-atividade e pensamento otimista determinam a integração e a co-criação de valor de um grupo, incluir atividades como palestras motivacionais podem aumentar o valor co-criado.

A mudança de comportamento é um ato voluntário, pois o ambiente institucional e os símbolos também direcionam recursos tanto para a mudança quanto para o reforço da manutenção do novo comportamento. As novas regras, sanções e garantias tendem a manter o novo comportamento e, portanto, criar uma identidade, o fato e o sentimento de pertencer àquilo que nos pertence.

Assim cumpre-se com o objetivo deste artigo teórico, de buscar resgatar a literatura de lazer como catalizador da transformação, fornecendo melhoria do bem-estar e das práticas de aprendizagem por meio da mudança de comportamento.

Profundamente ligados as questões Sociais, de Educação e de Formação Cidadã, a intenção de propor pressupostos basilares à esta discussão, são elencados a seguir, e merecem ser ampliados, como sugestão de novos estudos, que promovam a possibilidade de mensurar $\mathrm{o}$ impacto do lazer em ambientes de laborais como ferramenta de aprendizagem:

- $1^{o}$ Proposição: compreender agentes e entidades de serviço, de consumo e de produção do lazer - o macro ambiente que promove resultados para o bem-estar que priorizem a otimização, acessibilidade, comunicação apropriada, contribuir para captar o nível de sensibilidade aos desejos e necessidades.

- $2^{o}$ Proposição: envolver os agentes construção de uma capacidade que permita a transformação, focada em Mudança cognitiva, Mudança de Ação, Mudança no Comportamento e a Mudança de Valores.

- $\quad 3^{o}$ Proposição: conscientizar a existência da opção de mudança; aceitação dos valores que tornam a opção aceitável para adoção; percepção da opção como relevante para si, para familiares e para a sociedade; percepção de que as consequências positivas da opção superam as consequências negativas; crença de que se possui habilidade e capacidade para adoção da opção; crença de que pessoas consideradas importantes (família, amigos) que apoiam a adoção da opção.

- $4^{o}$ Proposição: Utilizar principalmente as premissas que Lazer pode ser a chave para trocas indiretas que co-criando atores sociais e econômicos são integradores de recursos; o valor é unicamente e fenomenologicamente determinado pelo beneficiário.

- $5^{\circ}$ Proposição: Mensurar e usar a capacidade na qual a proposta de um determinado comportamento cria novas situações de bem estar, considerando a eficiência, a eficácia e também a efetividade do processo de aprendizagem (análise de impactos em médio e longo prazo). 
Assim, através deste levantamento teórico, percebe-se o lazer é uma dimensão da cultura, do cotidiano social que, além de direito, é formado pela visão funcionalista como agente capacitor, formador e contribuinte da aprendizagem e bem estar dos indivíduos em diversos ambientes.

\section{REFERENCIAS}

Anderson Laurel (2010). "Improving Well-Being through Transformative Service," in "Moving Forward and Making a Difference: Research Priorities for the Science of Service," by Ostrom Amy L., Bitner Mary Jo, Brown Stephen W., Burkhard Kevin A., Goul Michael, Smith-Daniels Vicki, Demirkan Haluk, Rabinovich Elliott, Journal of Service Research, 13 (1), 4-36.

Anderson Laurel, Ostrom Amy L., Corus Canan, Fisk Raymond P., Gallan Andrew S., Giraldo Mario, Mende Martin, Mulder Mark \& Rayburn Steven W., Rosenbaum Mark S., Shirahada Kunio, Williams Jerome D. (2013), “Transformative Service Research: An Agenda for the Future," Journal of Business Research, 66 (8), 1203-1210.

Anderson, L., Ostrom, A. L., \& Bitner, M. J. (2011). Surrounded by services: A new lens for examining the influence of services as social structures on wellbeing. Working study, WP Carey School of Business, Arizona State University, Phoenix, AZ.

Bauman, Z. (2009). Confiança e medo na cidade. Rio de Janeiro: Zahar.

Bramante, Antonio Carlos (1998). Lazer: concepções e significados. Licere. Belo Horizonte, v.1, n.1. p.917.

Camargo, Luiz Octávio L (1986). O que é lazer. São Paulo: Brasiliense.

Dumazedier, Joffre (1973). Lazer e cultura popular. São Paulo: Perspectiva.

(1979). Sociologia empírica do lazer. São Paulo: Perspectiva.

Ferreira, Acácio (1959). Lazer Operário: Um estudo de organização social das cidades. Salvador: Livraria Progresso.

Fisk, Raymond (2009), “A Customer Liberation Manifesto,” Service Science, 1 (3), 135-141.

Foote, J., Baker, V., Carswell, S., Fa'asalele Tanuvasa, A., Finsterwalder, J., Hepi, M., \& Taylor, A. (2014). Towards a Service Ecology Approach to
Improve Social Service Uptake and Outcomes for the 'Hard to Reach'Populations. In Proceedings of the 58th Meeting of the International Society for the Systems Sciences-Learning Across Boundaries: Exploring the Variety of Systemic Theory and Practice, Washington DC.

GOMES, Christianne L. (2004). Verbete Lazer Concepções. In: GOMES, Christianne L. (Org.). Dicionário Crítico do Lazer. Belo Horizonte: Autêntica Editora. p.119-126.

(2003). Lazer e trabalho no contexto urbano: Reflexões sobre os "Clubes de Menores Operários” (1937-1947). In: MÜLLER, Ademir; DACOSTA, Lamartine P. (Org.). Lazer e trabalho: Um único ou múltiplos olhares? Santa Cruz do Sul/RS: EDUNISC. p. 27-44.

(2003). Significados de recreação e lazer no Brasil: Reflexões a partir da análise de experiências institucionais no âmbito das políticas públicas (1926-1964). Belo Horizonte: Faculdade de Educação/UFMG (Tese, Doutorado em Educação).

Kotler, P. (1978). Marketing Para Organizações Que Não Visam Lucro. São Paulo: Atlas.

Maslow, A.H. (1987). Motivation and Personality. Harper \& Row, New York.

Leite, C. (2012). Cidades sustentáveis, cidades inteligentes: desenvolvimento sustentável num planeta urbano. Porto Alegre: Bookman.

Marcellino, Nelson C. (1987). Lazer e educação. Campinas: Papirus.

(1983). Lazer e humanização.

Campinas: Papirus.

(2009).. Lazer, saúde e educação física: a corporeidade e a qualidade de vida. In: Moreira, W. W.; Simões, R. (Org.). Educação Física e produção de conhecimento. Belém: EDUFPA. v.1, p. 81-108.

(2011). Pedagogia da

animação. 10. ed. Campinas: Papirus.

Marinho, Inezil Penna (1957). Educação física, recreação e jogos. São Paulo: Cia Brasil Editora.

Mascarenhas, Fernando (2001). Lazer e trabalho: Liberdade ainda que tardia. In: Seminário "O lazer em debate”, 2, 2001, Belo Horizonte. Coletânea... Belo Horizonte: Imprensa Universitária/CELAR/DEF/UFMG. p.81-93. 
Mick, D.G., Pettigrew, S., Pechmann, C., Ozanne, J.L. (2012). Origins, qualities, and envisionments of transformative consumer research. In: Mick, D.G., Pettigrew, S., Pechmann, C., Ozanne, J. (Eds.), Transformative Consumer Research for Personal and Collective Well-Being. Routledge, New York, London, pp. 3-24.

Requixa, Renato (1977). O lazer no Brasil. São Paulo: Brasiliense.

(1980). Sugestão De Diretrizes

Para Uma Política Nacional De Lazer. São Paulo:

Sesc.

Rosenbaum, M. S., Corus, C., Ostrom, A. L., Anderson, L., Fisk, R. P., Gallan, A. S., ... Williams, J. D. (2011). Conceptualisation and aspirations of transformative service research. Journal of Research for Consumers, 19,1-6.

Ryan, R.M., Deci, E.L. (2001). On happiness and human potentials: a review of research onhedonic and eudaimonic well-being. Annual Reviews of Psychology. 52 (1), 141-166.

Santos, M. (2003). Por uma outra globalização: do pensamento único à consciência universal. Rio de Janeiro: Record.

Sussekind, Arnaldo, Marinho, Inezil P., Góes, Oswaldo (1952). Manual de Recreação (Orientação dos lazeres do trabalhador). Rio de Janeiro: Ministério do Trabalho, Indústria e Comércio.

Vargo, S.L., Lusch, R.F. (2006). Service-dominant logic: what it is, what it is not, what it might be. In: Lusch, R.F., Vargo, S.L. (Eds.), The ServiceDominant Logic of Marketing: Dialog, Debate, and Directions. ME Sharpe, Armonk, NY, p. 43-56.

Service-dominant logic:

continuing the evolution. Journal Academic of Marketing Science. 36 (1), 2008a. p.1-10.

(2008). Why "service"?.

Journal of the Academy of marketing Science, 36(1), 25-38.

(2011). It's all B2B... and

beyond: Toward a systems perspective of the market. Industrial marketing management, 40(2), 181-187.

(2008). Foundations \& frontiers of service-dominant logic. Paper presented at the Forum on Markets and Marketing, Karlstad, Sweden.

Vargo, S. L., Maglio, P. P., \& Akaka, M. A. (2008).

On value and value co-creation: A service systems and service logic perspective. European management journal, 26(3), 145-152.

Werneck, Christianne Luce Gomes (2000). Lazer, trabalho e educação: Relações históricas, questões contemporâneas. Belo Horizonte: Editora UFMG/CELAR.

Wieland, H., Polese, F., Vargo, S. L., \& Lusch, R. L. (2012). Toward a Service (Eco)Systems Perpective on value creation. International Journal of Service Science, Management, Engineering and Technology, 3(3), 12-25.

Williams, Jerome D. \& Geraldine R. Henderson (2012), "Discrimination and Injustice in the Marketplace: They Come in All Sizes, Shapes, and Colors," in David Mick, Simone Pettigrew, Connie Pechmann, and Julie Ozanne, eds. Transformative Consumer Research for Personal and Collective Well Being: Reviews and Frontiers, Boca Raton, FL: Taylor \& Francis, 171-190.

Aldair Fernandes Silva

Instituto Federal de Minas Gerais - IFMG.

Mestre em Administração.

Ricardo Soares de Melo

Centro Universitário Internacional - Uninter.

Mestre em Contabilidade.

Estudante de Licenciatura em Matemática.

\section{Ítalo Brener de Carvalho}

Centro Federal de Educação Tecnológica de Minas

Gerais - CEFET-MG.

Doutor em Administração.

Mestre em Negócios Internacionais. 